\title{
The Effect of Market Factor on Portfolio Selection
}

\author{
Daniel Arficho Gaenore ${ }^{1 *}$, M Montaz Ali $^{2}$, Aduda Jane Akinyi ${ }^{3}$ \\ ${ }^{1}$ Department of Statistics and Actuarial Sciences, Faculty of mathematics, Pan African University for Basic Sciences, \\ Technology and Innovations, Nairobi, Kenya \\ ${ }^{2}$ School of Computer Science and Applied Mathematics, University of the Witwatersrand, Johannesburg, South Africa \\ ${ }^{3}$ Department of Statistics and Actuarial Sciences, Faculty of mathematics, JKUAT, Nairobi, Kenya
}

\begin{abstract}
Received November 18, 2021; Revised January 21, 2022; Accepted February 8, 2022
Cite This Paper in the following Citation Styles

(a): [1] Daniel Arficho Gaenore, M Montaz Ali, Aduda Jane Akinyi, "The Effect of Market Factor on Portfolio Selection," Universal Journal of Accounting and Finance, Vol.10, No.2, pp. 624-634, 2022. DOI: 10.13189/ujaf.2022.100227
\end{abstract}

(b): Daniel Arficho Gaenore, M Montaz Ali, Aduda Jane Akinyi, (2022). The Effect of Market Factor on Portfolio S election. Universal Journal of Accounting and Finance, 10(2), 624-634. DOI: 10.13189/ujaf.2022.100227

Copyright $\odot 2022$ by authors, all rights reserved. Authors agree that this article remains permanently open access under the terms of the Creative Commons Attribution License 4.0 International License

\begin{abstract}
Investors' attempt is to allocate scarce resources today and receive the best outcome in future. The best outcome in future is not known today. But one can forecast for the best outcome in future today. Markowitz developed mean-variance model to solve portfolio selection problem. This model has two main drawbacks. The first is that the model assumes future expected return is the same as current expected return. In practice, this assumption may not hold in general. The second drawback is that the model requires a lot of estimations when investors consider large number of assets to include in portfolio. Sharpe developed single index model to solve the second drawback of mean-variance model. In this research, we develop future portfolio model to deal with drawbacks of mean-variance model. The objective of future portfolio model is to maximize future Sharpe ratio forecast subject to no short and no leftover constraints. This model is a realistic model because investors would like to consider future outcome instead of current outcome. First, we construct future expected return forecast model to forecast future expected return of securities. Second, we apply capital asset pricing model to estimate variance of portfolio. Third, we build future portfolio model using future expected return forecast model and capital asset pricing model. Fourth, we solve for analytic solution of future portfolio model. Finally, we evaluate the performance of future portfolio model relative to single index portfolio based on Sharpe ratio process metric, diversification ratio process metric and accumulation factor error process metric. The result of this study shows that future portfolio outperforms single index portfolio based on Sharpe ratio process metric, diversification ratio process metric and accumulation factor error process metric except at few instant time points.
\end{abstract}

Keywords Accumulation Factor, Arbitrage, Forecast,
Model, Sharpe Ratio

\section{Introduction}

Markowitz realized that risk and return are positively correlated. He noticed that for every given level of risk there is corresponding optimal level of return. However, investors do not know the actual future return and risk. Investors choose a portfolio from efficient frontier based on their risk tolerance. Mean variance portfolio is a portfolio that can be chosen from efficient frontier using targeted level of risk. Since mean variance portfolio depends on estimated value of expected return, variance of return and covariance returns, it contains parameters' estimation error. Minimum variance portfolio is a portfolio which is constructed by minimizing the variance of portfolio. Researchers have done empirical study to evaluate the performance of mean variance and minimum variance portfolio out of sample. Empirical studies show that mean variance portfolio is very unstable and performs poorly out of sample in terms of Sharpe ratios [1]. Minimum variance portfolio is less susceptible to the estimation error than mean variance portfolio, [1]. Minimum variance portfolio performs better out of sample relative to mean variance portfolio in terms of mean and variance, [1]. Equally weighted portfolio is a portfolio which contains equal weight for each security in it. Empirical evidence shows that equally weighted portfolio outperforms mean variance portfolio and minimum variance portfolio, [1]. Mean variance portfolio lacks diversification and shows poor out of sample performance, [2]. Markowitz's celebrated meanvariance portfolio optimization theory assumes that the means and covariances of the underlying asset returns are known, [3]. 
In practice, they are unknown and have to be estimated from historical data, [3]. Estimation of the expected returns and the covariances for the financial assets has a significant importance in quantitative portfolio management, [4]. The famous financial models used in estimating the input parameters is CAPM, [4]. The performances of optimized CAPM portfolios are higher than multi-factor models, [4]. Asset pricing models have significant role in the Markowitz's mean-variance optimization technique since they provide higher portfolio performances with lower risks than the optimized portfolios of naive returns, [4]. The attraction of CAPM is that it offers powerful and intuitively pleasing predictions about how to measure risk and the relation between expected return and risk, [5]. We have described some drawbacks of mean variance portfolio.

It is clear that CAPM uses historical data not the estimated future forecast. In this research, we use historical data, future estimated expected return and future estimated variance to construct future portfolio. We do not rely on historical data only but we also consider estimated future expectation and risk to construct portfolio. For this reason, researchers are interested to forecast future expected return to construct portfolio. Sharpe constructed single index model using capital asset pricing model to simplify the mean-variance portfolio model. Single index model does not consider future expected return forecast. But investors want to consider future expected return forecast for portfolio selection. This motivates researchers to study portfolio selection using future expected return forecast. In this research, we contribute two novel models for portfolio selection. The first model is future expected return forecast model. This model is very important for forecasting future return of securities. It is important for constructing future portfolio model. The second model is future portfolio model. This model is important for investors who want to construct portfolio by considering future expected return forecast of securities. Our empirical study shows that future portfolio is outperforming equally weighted portfolio in the short run and single index portfolio based on Sharpe ratio process. Moreover, this future portfolio model requires $4 n+1$ estimation of portfolio parameters for $n$ number of securities. We know that mean-variance model requires $\frac{n^{2}+n}{2}+n$ estimation of portfolio parameters for $n$ number of securities. This shows that future portfolio is less complex than mean-variance portfolio for large number of securities. Thus, we notice that future portfolio model is complex than mean-variance portfolio model. It is clear that single index model requires $3 n+1$ estimation of portfolio parameters for $n$ number of securities. This shows that single index model is more simple than future portfolio model. But single index model does not include future expected return forecast for portfolio construction.

In finance, portfolio selection models are very important for investors and practitioners. Past historical data helps investors when they want to do technical analysis. There are a lot of future return forecasting methods. In this research, we propose to build future expected return forecast model and future portfolio model to construct future portfolio.

Investors want to use realistic model to construct portfolio. Mean-variance model and single index model assumes that future expected return is the same as current expected return.
This assumption does not work for general case. The problem we would like to solve is constructing future portfolio using future portfolio model.

The general objective of this study is to construct future portfolio using future portfolio model. First, we build future expected return forecast model to estimate future expected return and apply capital asset pricing model to estimate current portfolio variance. Second, we construct future portfolio model and solve future portfolio model analytically. Finally, we evaluate future portfolio performance relative to equally weighted portfolio, single index portfolio and market index portfolio based on Sharpe process.

\section{Literature review}

Investor should allocate financial resources in efficient and effective way to maximize return as well as to minimize investment cost. Constructing an optimal portfolio is allocating financial or investment resources in effective and efficient way to maximize portfolio return and to minimize portfolio risk.Investment definition simple. Refer [6,7]. for investment definition. Risk is defined briefly in $[7,8]$. Portfolio selection models depend on investors' risk preference. See $[9,10,11,13]$ for definition of risk preference, risk tolerance and risk aversion. Some basic pordolio selection models are risk neutral Markowitz's model [14], return neutral Markowitz's model, risk adjusted model [15], Sharpe ratio maximization model $[16,17]$, diversification maximization model and Treynor ratio maximization model. SharpeLintner Capital Asset Pricing Model (CAPM) was introduced by Sharpe [1964] and Lintner [1965], [18]. Researchers, Macbeth, Fama, Friend and Bloom, confirmed CAPM in 1973, [19]. However,Cheng, Grower and Gibbons tested CAPM and rejected it, [19]. [20] tested that Alphas for a majority of the Nairobi Securities Exchange stocks are significantly different from zero. A portfolio is called tangent portfolio if it is constructed by Sharpe ratio maximization model, [21]. Sharpe's single index model is the easiest and the most widely used model in constructing an optimal portfolio. Researchers have used cut off point to determine the optimal portfolio using single index model, $[21,22,23]$. The review of many portfolio performance measures is given in,[24]. In [25], the author mentioned some suitable portfolio measures for different portfolios. 17]:

Sharpe ratio maximization model (SRMM) is given by, [16,

$$
\begin{array}{ll}
(S R M M) & \max _{w} \frac{\sum_{i=1}^{n}\left(\mu_{i}-r_{f}\right) w_{i}}{\sqrt{\sum_{i=1}^{n} \sum_{j=1}^{n} \sigma_{i j} w_{i} w_{j}}} \\
\text { subject to } & \sum_{i=1}^{n} w_{i}=1, \\
& 0 \leq w_{i} \leq 1, \forall i=1,2, \ldots, n .
\end{array}
$$

Single index model(SIM) assumes that return of security as a linear function of market return. Furthermore, we assume that $\operatorname{cov}\left(e_{i}, e_{j}\right)=0$ for $i \neq j$ and $\operatorname{cov}\left(r_{m}, e_{i}\right)=0$ for all $i$. 
Mathematically, we describe return of security $i$ as follows:

$$
r_{t i}-r_{f}=a_{i}+b_{i}\left(r_{t m}-r_{f}\right)+e_{t i} .
$$

One can estimate parameters $a_{i}$ and $b_{i}$ as follows: $\hat{b}_{i}=$ $\frac{\operatorname{cov}\left(r_{t i}, r_{t m}\right)}{\operatorname{var}\left(r_{t m}\right)}$ and $\hat{a}_{i}=\mu_{i}-r_{f}-\hat{b}_{i}\left(\mu_{m}-r_{f}\right)$, [18]. Single index model is given by [18]:

$$
\begin{aligned}
\text { SIM } & \max _{w} \frac{\sum_{i=1}^{n}\left(\mu_{i}-r_{f}\right) w_{i}}{\sqrt{\left(\sum_{i=1}^{n} \hat{b_{i}} w_{i}\right)^{2} \sigma_{m}^{2}+\sum_{i=1}^{n} \sigma_{e_{i}}^{2} w_{i}^{2}}} \\
\text { subject to } & \sum_{i=1}^{n} w_{i}=1, \\
& 0 \leq w_{i} \leq 1, \quad \forall i=1,2, \ldots, n .
\end{aligned}
$$

\section{Methodology}

This research presents portfolio selection technique based on market factor and future expected return and variance. We introduce a new future return forecasting method to construct portfolio based on market factor and future expected return. Furthermore, we show the derivation of future expected return in this chapter. Moreover, we evaluate the performance of single index portfolio and future portfolio using Sharpe process.

Definition 3.1 (Sharpe ratio process). Note that Sharpe ratio process (vector) is defined by $s r_{t p}=$ $\left(\frac{\mu_{2 p}-r_{f}}{\sigma_{2 p}}, \frac{\mu_{3 p}-r_{f}}{\sigma_{3 p}}, \ldots, \frac{\mu_{t p}-r_{f}}{\sigma_{t p}}\right)^{T}$.

Definition 3.2. Beta of security $i$ is given by $\beta_{i}=\frac{\sigma_{i m}}{\sigma_{m}^{2}}$, where $\sigma_{m}^{2}$ is variance of market index and $\sigma_{i m}$ is co-variance between security $i$ and market index.. Alpha of security $i$ is given by $\alpha_{i}=\mu_{i}-r_{f}-\frac{\sigma_{i m}}{\sigma_{m}^{2}}\left(\mu_{m}-r_{f}\right)$, where $\mu_{i}$ is mean of security $i$, $\mu_{m}$ is mean of market index $m$ and $r_{f}$ is risk free rate of return.

Definition 3.3. A security $i$ is called under performing (overpriced or overvalued) security if $\alpha_{i}<0$.

Investors would like to sell under performing securities. They prefer to buy undervalued securities or fairly priced securities. Suppose that there are $m$ number of under performing securities. Let $n$ be total number of securities. Then split the return matrix $R M$ of securities into two matrices $R M_{u}$ and $R M_{c}$. A matrix $R M_{u}$ is called return matrix of under performing securities. $R M_{C}$ is called return matrix of outperforming or fairly priced securities. Note that the number of columns of $R M_{u}$ is $m$, and the number of columns of $R M_{o}$ is $n-m$.

Let us consider no arbitrage model $(N A M)$. Artificial short selling is assuming that borrowing from under performing companies is allowed in a sense that an investor has capital which is equivalent to borrowed capital. That is, an investor who invested $w_{o}$ capital in under performing companies can borrow $w_{o}$. Note that an investor who invests $w_{o}$ in under performing companies has second alternative investment opportunity. This opportunity is investing in outperforming or fairly valued companies. Thus, investors can use artificial short selling to get zero opportunity cost. They borrow from under performing companies and invest the borrowed capital in outperforming or fairly priced companies. However, this attempt should give zero net expected return and zero net variance. That is, there is no arbitrage opportunity. Net expected return is $n e_{r}=\sum_{i=m+1}^{n} \mu_{i} w_{i}-\sum_{i=1}^{m} \mu_{i} w_{i}$. Define

$$
\begin{gathered}
a=\sum_{i=m+1}^{n} \sum_{j=m+1}^{n} \operatorname{cov}\left(r_{i}, r_{j}\right) w_{i} w_{j} . \\
b=\sum_{i=1}^{m} \sum_{j=1}^{m} \operatorname{cov}\left(r_{i}, r_{j}\right) w_{i} w_{j} . \\
c=\sum_{i=m+1}^{n} \sum_{j=1}^{m} \operatorname{cov}\left(r_{i}, r_{j}\right) w_{i} w_{j} .
\end{gathered}
$$

Similarly, net variance is defined by:

$$
n e_{v}=a+b-2 c .
$$

Thus, no arbitrage model $(N A M)$ is given by:

$$
\begin{aligned}
N A M: & \min _{w} n e_{r}^{2} \\
\text { Subject to }: & \sum_{i=1}^{m} w_{i}=\sum_{i=m+1}^{n} w_{i}=1, \\
& n e_{v}=0, \\
& 0 \leq w_{i} \leq 1, \quad \forall i=1,2, \ldots, n .
\end{aligned}
$$

The above problem, $N A M$, is solved to obtain $w=$ $\left(w_{1}, w_{2}, \ldots, w_{n}\right)^{T}$. The resulting portfolio is called market arbitrage portfolio $(M A P)$.

In finance literature, portfolio accumulation factor constraint is not known. However, portfolio accumulation factor constraint is a logical constraint that investors should consider to construct portfolio. Let us introduce portfolio accumulation factor constraint. Let $v_{\tau}$ be the portfolio value at time $\tau$. Then there exists a unique portfolio accumulation factor $a_{\tau}$ such that $v_{\tau}=v_{1} a_{\tau}$, where $v_{1} \neq 0$ is principal value of portfolio.

Definition 3.4 (Accumulation factor constraint lemma). The accumulation factor of portfolio of $n$ securities over period $[2, \tau]$ is defined by:

$$
a_{\tau}=\sum_{j=1}^{n}\left(\prod_{t=2}^{\tau}\left(1+r_{t j}\right)\right) w_{j}=\prod_{t=2}^{\tau} \sum_{j=1}^{n}\left(1+r_{t j}\right) w_{j} .
$$

Define

$$
\begin{gathered}
e_{1 \tau}=\sum_{j=1}^{n}\left(\prod_{t=2}^{\tau}\left(1+r_{t j}\right)\right) w_{j} . \\
e_{2 \tau}=\prod_{t=2}^{\tau} \sum_{j=1}^{n}\left(1+r_{t j}\right) w_{j} .
\end{gathered}
$$

Note that $e_{a_{\tau}}=\left(e_{1 \tau}-e_{2 \tau}\right)^{2}$ is called accumulation factor error.

Definition 3.5 (Diversification ratio process). Define

$$
r_{t i}^{* *}=\left(r_{1 i}, r_{2 i}, \ldots, r_{t i}\right)^{T} .
$$




$$
d s_{t}=\frac{\sum_{i=1}^{n} \sqrt{\operatorname{variance}\left(r_{t i}^{* *}\right)} w_{i}}{\sqrt{\sum_{i=1}^{n} \sum_{j=1}^{n} \operatorname{cov}\left(r_{t i}^{* *}, r_{t j}^{* *}\right) w_{i} w_{j}}} .
$$

Diversification ratio process is defined by:

$$
d p_{\tau p}=\left(d s_{2}, d s_{3}, \ldots, d s_{\tau}\right)^{T} .
$$

Definition 3.6 (Accumulation factor error process). Асcumulation factor error process is defined by: afep $p_{\tau p}=$ $\left(e_{a_{2}}, e_{a_{3}}, \ldots, e_{a_{\tau}}\right)^{T}$.

Definition 3.7 (Total error). Total error of portfolio is defined by: $t e_{p}=\sum_{t=2}^{\tau} e_{a_{t}}$.

Definition 3.8 (Total reward error ratio). Total reward error ratio is defined by: trer $_{p}=\sum_{t=2}^{\tau} \frac{\mu_{t p}-r_{f}}{\sqrt{e_{a_{t}}}}$.

Definition 3.9 (Reward error ratio process). Reward error ratio process is defined by : $\operatorname{rerp}_{p}=$ $\left(\frac{\mu_{2 p}-r_{f}}{\sqrt{e_{a_{2}}}}, \frac{\mu_{3 p}-r_{f}}{\sqrt{e_{a_{3}}}}, \ldots, \frac{\mu_{\tau p}-r_{f}}{\sqrt{e_{a_{\tau}}}}\right)^{T}$.

Portfolio accumulation factor constraint contains sums and products of returns and weighs. It looks difficult for solving portfolio construction models. Thus, we assume portfolio return is constant approximately to simplify portfolio accumulation factor constraint. Consider constant portfolio model (CPM). We know that $\sum_{j=1}^{n}\left(\prod_{t=2}^{\tau}\left(1+r_{t j}\right)\right) w_{j}=$ $\prod_{t=2}^{\tau} \sum_{j=1}^{n}\left(1+r_{t j}\right) w_{j}$. This implies

that $\frac{d\left(\sum_{j=1}^{n}\left(\prod_{t=2}^{\tau}\left(1+r_{t j}\right)\right) w_{j}\right)}{d w_{j}}=\frac{d\left(\prod_{t=2}^{\tau}\left(\sum_{j=1}^{n}\left(1+r_{t j}\right) w_{j}\right)\right)}{d w_{j}}$. The equation $e_{a_{\tau}}(P)=0$ is called accumulation factor constraint of portfolio $P$. Constant portfolio model is given by:

$$
\begin{aligned}
C P M: & \sum_{j=1}^{n} w_{j}=1 \\
& \frac{d e_{1 \tau}}{d w_{j}}=\frac{d e_{2 \tau}}{d w_{j}} \\
& \sum_{j=1}^{n} r_{t j} w_{j}=r_{t c}=r_{\tau c} \forall t=2,3, \ldots, \tau \text { and } t \geq 3
\end{aligned}
$$

Even though the assumption of portfolio return is constant approximately simplifies portfolio accumulation factor constraint it has disadvantage which is portfolio return may not be constant over time.

Definition 3.10. Let $f_{t}(x)$ be a non-zero differentiable function of $x \forall t=1,2, \ldots, \tau$. Then

$$
\frac{d \prod_{t=1}^{\tau} f_{t}(x)}{d x}=\left(\prod_{t=1}^{\tau} f_{t}(x)\right)\left(\sum_{t=1}^{\tau} \frac{\frac{d f_{t}(x)}{d x}}{f_{t}(x)}\right) .
$$

Consider the optimal solution of constant portfolio model (CPM). Clearly,

$$
\frac{d\left(\sum_{j=1}^{n}\left(\prod_{t=2}^{\tau}\left(1+r_{t j}\right)\right) w_{j}\right)}{d w_{j}}=\prod_{t=2}^{\tau}\left(1+r_{t j}\right) .
$$

Use Definition 3.10 to find equation (20).

$$
\frac{d e_{2 \tau}}{d w_{j}}=\left(\sum_{t=2}^{\tau} \frac{\left(1+r_{t j}\right)}{\sum_{j=1}^{n}\left(1+r_{t j}\right) w_{j}}\right) e_{2 \tau} .
$$

Since $r_{\tau c}=\sum_{j=1}^{n}\left(1+r_{t j}\right) w_{j}$, it follows from equation (20) that

$$
\frac{d e_{2 \tau}}{d w_{j}}=\left(\frac{\sum_{t=2}^{\tau}\left(1+r_{t j}\right)}{\sum_{j=1}^{n}\left(1+r_{t j}\right) w_{j}}\right) e_{2 \tau} .
$$

This implies that

$$
\frac{d e_{2 \tau}}{d w_{j}}=\left(\sum_{t=2}^{\tau}\left(1+r_{t j}\right)\right)\left(\prod_{t=2}^{\tau-1}\left(\sum_{j=1}^{n}\left(1+r_{t j}\right) w_{j}\right)\right) .
$$

Clearly,

$$
\frac{d e_{2 \tau}}{d w_{j}}=\left(\sum_{t=2}^{\tau}\left(1+r_{t j}\right)\right)\left(\prod_{t=2}^{\tau-1}\left(1+r_{\tau c}\right)\right) .
$$

This implies that

$$
\frac{d e_{2 \tau}}{d w_{j}}=\left(1+r_{\tau c}\right)^{\tau-2} \sum_{t=2}^{\tau}\left(1+r_{t j}\right) .
$$

It follows from equations 19 and 24 that

$$
1+r_{\tau c}=\left(\frac{\prod_{t=2}^{\tau}\left(1+r_{t j}\right)}{\sum_{t=2}^{\tau}\left(1+r_{t j}\right)}\right)^{\frac{1}{\tau-2}} .
$$

Set $u_{\tau j}=\frac{\prod_{t=2}^{\tau}\left(1+r_{t j}\right)}{\sum_{t=2}^{\tau}\left(1+r_{t j}\right)}$. Then $1+r_{\tau c}=\left(\frac{\sum_{j=1}^{n} u_{\tau j}}{n}\right)^{\frac{1}{\tau-2}}$. This implies that $r_{\tau c}=\left(\frac{\sum_{j=1}^{n} u_{\tau j}}{n}\right)^{\frac{1}{\tau-2}}-1$.

Thus, $r_{t c}=\left(\frac{\sum_{j=1}^{n} u_{t j}}{n}\right)^{\frac{1}{t-2}}-1 \forall t=3,4, \ldots, \tau$. Since $\sum_{j=1}^{n} r_{t j} w_{j}=r_{t c}$, we obtain $\sum_{j=1}^{n} r_{t j} w_{j}=$ $\left(\frac{\sum_{j=1}^{n} u_{t j}}{n}\right)^{\frac{1}{t-2}}-1 \forall t=3,4, \ldots, \tau$. Now, one can solve for $w_{j}$ from the following error sum of square minimization model.

$\operatorname{ESMM}: \min _{w} \sum_{t=3}^{\tau}\left(\left(\frac{\sum_{j=1}^{n} u_{t j}}{n}\right)^{\frac{1}{t-2}}-1-\sum_{j=1}^{n} r_{t j} w_{j}\right)^{2}$

Subject to : $\sum_{j=1}^{n} w_{j}=1$,

$$
0 \leq w_{j} \leq 1, \forall j=1,2, \ldots, n .
$$

We do not consider only a single time instant point for accumulation factor error. We evaluate the performance of portfolios using total error. Let us consider total error minimization model. Total error minimization model (TEMM) is given by:

$$
\begin{aligned}
& \text { TEMM: } \min _{w} t e_{p} \\
& \text { Subject to : } \\
& \qquad \sum_{j=1}^{n} w_{j}=1, \\
& 0 \leq w_{j} \leq 1, \forall j=1,2, \ldots, n .
\end{aligned}
$$


The optimal portfolio obtained from TEMM is called minimum error portfolio.

Portfolio Sharpe ratio requires a lot of parameters' estimation for large number of securities of the portfolio. We note that estimations contain errors. In this research, we argue that accumulation factor error risk measure is more realistic risk measure than variance risk measure. Portfolios should satisfy accumulation factor constraint. But in practice, minimizing accumulation factor error is good attempt to satisfy accumulation factor constraint approximately. Minimizing accumulation factor error penalizes return.

Some investors would like to construct diversified portfolio relative to accumulation factor error. Thus, we construct accumulation diversification model $(A D M)$. Accumulation diversification model $(A D M)$ is given as follows.

$$
\begin{aligned}
A D M: & \max _{w} \frac{\sum_{j=1}^{n} \sigma_{j} w_{j}}{\sqrt{e_{a_{\tau}}(p)}} \\
\text { Subject to }: & \sum_{j=1}^{n} w_{j}=1, \\
& 0 \leq w_{j} \leq 1, \forall j=1,2, \ldots, n .
\end{aligned}
$$

Note that accumulation diversification model requires $3 n$ parameters' estimation for $n$ number securities. The optimal portfolio constructed from accumulation diversification model is called accumulation diversification portfolio. There are some investors who want to minimize accumulation factor error. These investors can use accumulation factor error model (AFEM) to construct portfolio.

Accumulation factor error model (AFEM) is given by:

$$
\begin{aligned}
\text { AFEM : } & \min _{w} e_{a_{\tau}}(p) \\
\text { Subject to }: & \sum_{j=1}^{n} w_{j}=1, \\
& 0 \leq w_{j} \leq 1, \forall j=1,2, \ldots, n .
\end{aligned}
$$

Accumulation factor error model requires $2 n$ parameters' estimation for $n$ number securities. The optimal portfolio constructed from accumulation factor error model is called accumulation factor error portfolio.

Let us use second order Taylor series approximation for accumulation factor constraint. Apply natural logarithm on accumulation factor $a_{\tau}$ and express the result as second order Taylor series approximation. Note that second order Taylor series approximation of $\ln (x)$ is given by: $\ln (x) \approx x-1-\frac{1}{2}(x-1)^{2}$ provided $|x-1|$ is a small number. Clearly, $\left|a_{\tau}-1\right|$ is a small number. Thus, second order Taylor series approximation is appropriate for $\ln \left(a_{\tau}\right)$. Define accumulation factor vector $a \vec{f}_{n}$ by:

$$
a=\left(\prod_{t=1}^{\tau}\left(1+r_{t 1}\right), \prod_{t=1}^{\tau}\left(1+r_{t 2}\right), \ldots, \prod_{t=1}^{\tau}\left(1+r_{t n}\right)\right)^{T} .
$$

Clearly, $a_{p}=a^{T} w$.

Definition 3.11 (Second order accumulation factor constraint). Second order accumulation factor constraint is defined by:

$$
\left(a_{p}-2\right)^{2} \approx \tau\left(\mu_{p}-1\right)^{2}+(\tau-1)\left(\sigma_{p}^{2}-1\right) .
$$

Second order accumulation factor constraint is derived by the help of second order Taylor series approximation of $\ln \left(a_{p}\right)$. We note that portfolio variance can be determined approximately by three factors, namely, investment horizon, portfolio expected return and portfolio accumulation factor.

Mean variance portfolio theory states that portfolio variance as a function of portfolio expected return. However, Second order accumulation factor constraint tells us portfolio variance is not only a function of portfolio expected return but it is also a function of investment horizon and accumulation factor of portfolio approximately. Define

$$
\text { sov }=\left(\tau\left(\mu_{p}-1\right)^{2}+(\tau-1)\left(\sigma_{p}^{2}-1\right)-\left(a_{p}-2\right)^{2}\right)^{2} .
$$

The proposed second order accumulation factor index model is given below.

$$
\begin{aligned}
\text { SOAFIM : } & \max _{w} \frac{\mu_{p}-r_{f}}{\sqrt{s o v}} \\
\text { Subject to }: & \sum_{i=1}^{n} w_{i}=1, \\
& 0 \leq w_{i} \leq 1, \forall i=1,2, \ldots, n .
\end{aligned}
$$

second order accumulation factor index model requires $2 n+$ $\frac{n(n+1)}{2}$ parameters' estimation for $n$ number securities. The optimal portfolio constructed from second order accumulation factor index model is called second order accumulation factor index portfolio. Let us consider future portfolio model (FPM). We use future expected return forecast model and CAPM to construct future portfolio model. Let $t$ be time. Then note that

$$
\begin{gathered}
\mu_{(t+1) p}=\frac{\sum_{i=1}^{t+1} r_{i p}}{t+1}=\frac{t \mu_{t p}+r_{(t+1) m}}{t+1} . \\
\sigma_{(t+1) p}^{2}=\frac{t-1}{t} \sigma_{t p}^{2}+(t+1)\left(\mu_{(t+1) p}-\mu_{t p}\right)^{2} .
\end{gathered}
$$

Define

$$
\begin{gathered}
c_{0 t}=\left(a_{t p}^{2}-1\right) t^{2} \mu_{t p}^{2}+2 t \mu_{t p}\left(2 a_{t p}-1\right), \\
x_{2 t}=(t+1)^{2}\left(a_{t p}^{2}-1\right), \\
x_{1 t}=2(t+1)\left(1+t \mu_{t p}-\left(2 a_{t p}+t a_{t p}^{2} \mu_{t p}\right)\right), \\
\mu_{1 p}^{\delta}=\mu_{1 p} \\
\mu_{(t+1) p}^{\delta}=\frac{-x_{1 t}-\sqrt{x_{1 t}^{2}-4 x_{2 t} c_{0 t}}}{2 x_{2 t}} .
\end{gathered}
$$

Future expected return forecast model is given by:

$$
\mu_{t i}=a_{i}^{\delta}+b_{i}^{\delta} \mu_{t i}^{\delta}+e_{t i} .
$$

Future expected return forecast of security $i$ is given by:

$$
\mu_{(\tau+1) i}=a_{i}^{\delta}+b_{i}^{\delta} \mu_{(\tau+1) i}^{\delta} .
$$

Define future expected return forecast of portfolio $p$ by:

$$
\mu_{(\hat{\tau+1) p}}=\sum_{i=1}^{n}\left(a_{i}^{\delta}+b_{i}^{\delta} \mu_{(\tau+1) i}^{\delta}\right) w_{i} .
$$


Clearly,

$$
\sigma_{\tau p}^{2}=\left(\sum_{i=1}^{n} \hat{b_{i}} w_{i}\right)^{2} \sigma_{m}^{2}+\sum_{i=1}^{n} \sigma_{e_{i}}^{2} w_{i}^{2} .
$$

Define future variance of return forecast of portfolio $p$ by:

$$
{\sigma_{(\tau+1) p}^{2}}^{\wedge}=\frac{\tau-1}{\tau} \sigma_{\tau p}^{2}+(\tau+1)\left(\mu_{(\tau+1) p}-\mu_{\tau p}\right)^{2} .
$$

Consider future Sharpe ratio forecast $S R_{(\tau+1) p}$. $S R_{(\tau+1) p}=\frac{\mu_{(\tau \hat{+} 1) p}-r_{f}}{\sigma_{(\tau \hat{+} 1) p}}$.

Clearly,

Future portfolio model FPM is given by:

$$
\begin{aligned}
\text { FPM } & \max _{w} S R_{(\hat{\tau+1) p}} \\
\text { subject to } & \sum_{i=1}^{n} w_{i}=1, \\
& 0 \leq w_{i} \leq 1, \forall i=1,2, \ldots, n .
\end{aligned}
$$

The optimal portfolio constructed from future portfolio model is called future portfolio. We use the following symbols to find optimal solution of future portfolio model (FPM).

1. $g=1+\sigma_{m}^{2} \sum_{i=1}^{n}\left(\frac{\hat{\underline{b}}^{2}}{\sigma_{e i}^{2}}\right)$

2. $y_{i}=a_{i}^{\delta}+b_{i}^{\delta} \mu_{(\tau+1) i}^{\delta}-\mu_{\tau i}$,

3. $\theta_{i}=a_{i}^{\delta}+b_{i}^{\delta} \mu_{(\tau+1) i}^{\delta}-r_{f}$,

4. $h_{i}=\theta_{i}-\frac{\hat{b_{i}} \sigma_{m}^{2} \sum_{i=1}^{n}\left(\frac{\hat{b}_{i} \theta_{i}}{\sigma_{e i}^{2}}\right)}{g}$,

5. $f_{i}=(\tau+1)\left(y_{i}-\frac{\hat{b_{i}} \sigma_{m}^{2} \sum_{i=1}^{n}\left(\frac{\hat{b_{i}} y_{i}}{\sigma_{e i}^{2}}\right)}{g}\right)$,

6. $s=\frac{\sum_{i=1}^{n}\left(\frac{y_{i} h_{i}}{\sigma_{e i}^{2}}\right)}{\frac{\tau-1}{\tau}+\sum_{i=1}^{n}\left(\frac{y_{i} f_{i}}{\sigma_{e i}^{2}}\right)}$,

7. $z_{i}=\frac{\left(h_{i}-s f_{i}\right) \tau}{(\tau-1) \sigma_{e i}^{2}}$,

8. $k=\frac{\tau-1}{\tau}\left(\left(\sum_{i=1}^{n} \hat{b_{i}} z_{i}\right)^{2} \sigma_{m}^{2}+\sum_{i=1}^{n} \sigma_{e_{i}}^{2} z_{i}^{2}\right)+(\tau+$ 1) $\left(\sum_{i=1}^{n}\left(a_{i}^{\delta}+b_{i}^{\delta} \mu_{(\tau+1) i}^{\delta}-\mu_{\tau i}\right) z_{i}\right)^{2}$,

9. $x_{i}=\left\{\begin{array}{l}0 \text { if } \frac{r_{f} z_{i}}{\left(\sum_{i=1}^{n}\left(a_{i}^{\delta}+b_{i}^{\delta} \mu_{(\tau+1) i}^{\delta}\right) z_{i}\right)-k}<0 \\ \frac{r_{f} z_{i}}{\left(\sum_{i=1}^{n}\left(a_{i}^{\delta}+b_{i}^{\delta} \mu_{(\tau+1) i}^{\delta}\right) z_{i}\right)-k} \text { else }\end{array}\right.$.

The optimal solution of future portfolio model is given by:

$$
w_{i}^{*}=\frac{x_{i}}{\sum_{i=1}^{n} x_{i}} .
$$

\section{Results}

In this research, we use 01 Jan 2020- 22 Dec 2021 daily return historical data of USA 106 stock companies from yahoo finance. We also use one year bond rate $r_{f}=0.11 \%$ (daily bond rate $r_{f}=0.000003$ ) for empirical study. We apply capital asset pricing model to estimate current portfolio variance. Let us evaluate the performance of capital asset pricing model (CAPM) and future expected return forecast model for expected returns. Consider company $A M Z N$ and market index $S \& P 500$. Apply CAPM to estimate return of $A M Z N$. Then find corresponding expected return.

CAPM gives sum of square errors $\sum_{t=1}^{\tau}\left(\mu_{t}-\hat{\mu_{t}}\right)^{2}=$ 0.00076724 whereas our model future expected return forecast model gives $\sum_{t=1}^{\tau}\left(\mu_{t}-\hat{\mu_{t}}\right)^{2}=0.000105711$. This implies that CAPM sum of square errors is 625.7928556 percent more than future expected return forecast model sum of square errors. Therefore, we conclude that future expected return forecast model outperforms CAPM for expected return of $A M Z N$ company.

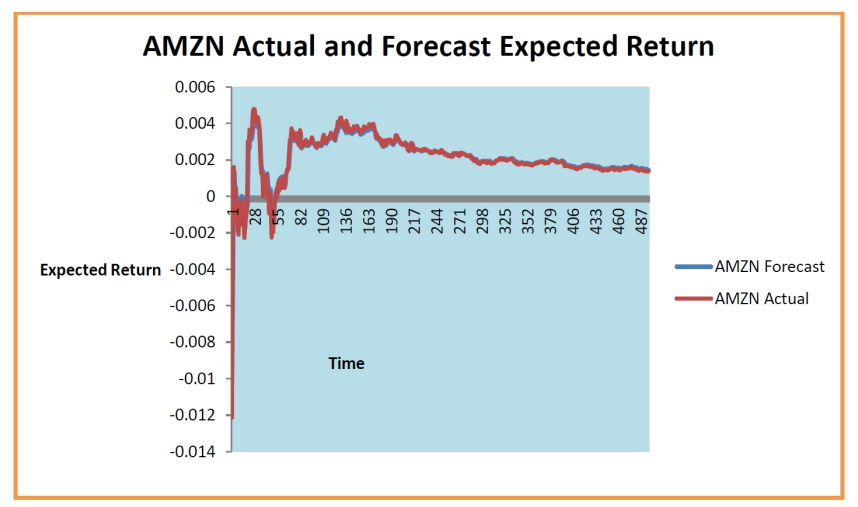

Figure 1. Company AMZN actual and forecast expected return

The above Figure 1 shows that the forecast of expected return of AMZN company follows actual expected return of AMZN company. Note that future expected return forecast model does not use market index return to forecast future expected return of securities. Security return estimation by CAPM requires return of market index. Note that from CAPM, future expected return of security depends on future expected return of market index. Since future expected return of market index is not known, future expected return of security is not known from CAPM. Future expected return forecast model has a potential to forecast future expected return from past historical return. Therefore, future expected return forecast model is better than CAPM relative to forecasting potential and estimation error.

There are a lot of portfolio performance measures in finance literature. In this research, we introduce new portfolio performance measures, namely, total error metric and total reward error ratio metric. The less total error portfolio is the more favorable portfolio. Note that the more total reward error ratio portfolio is the more favorable portfolio. In this research, we have constructed five new portfolios using historical data. These portfolios are accumulation factor error portfolio, sec- 
ond order accumulation factor index portfolio, future portfolio, accumulation diversification portfolio and minimum error portfolio. We evaluate the performance of these portfolios using Sharpe ratio process, diversification ratio process, accumulation factor error process, total error, total reward error ratio process and total reward error ratio measures. Furthermore, we evaluate the performance of single index portfolio and future portfolio using portfolio performance measures.

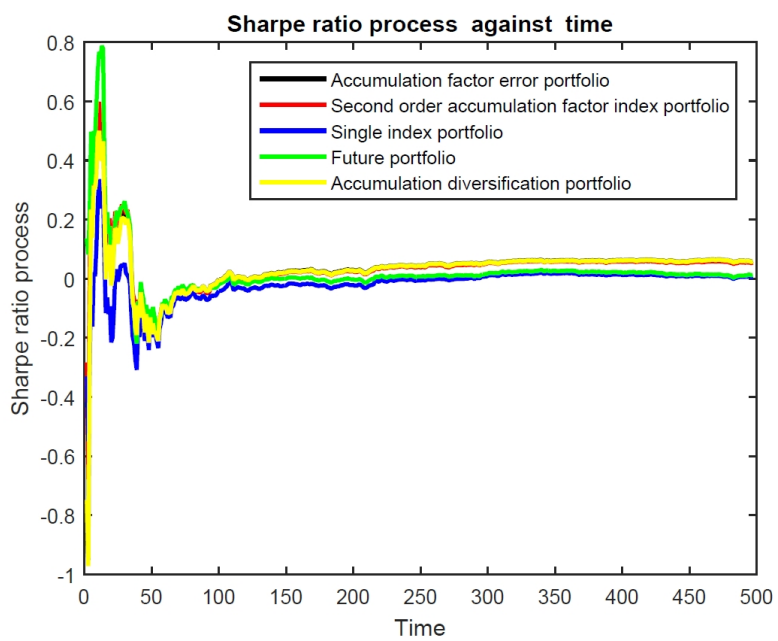

Figure 2. Sharpe ratio process of portfolios

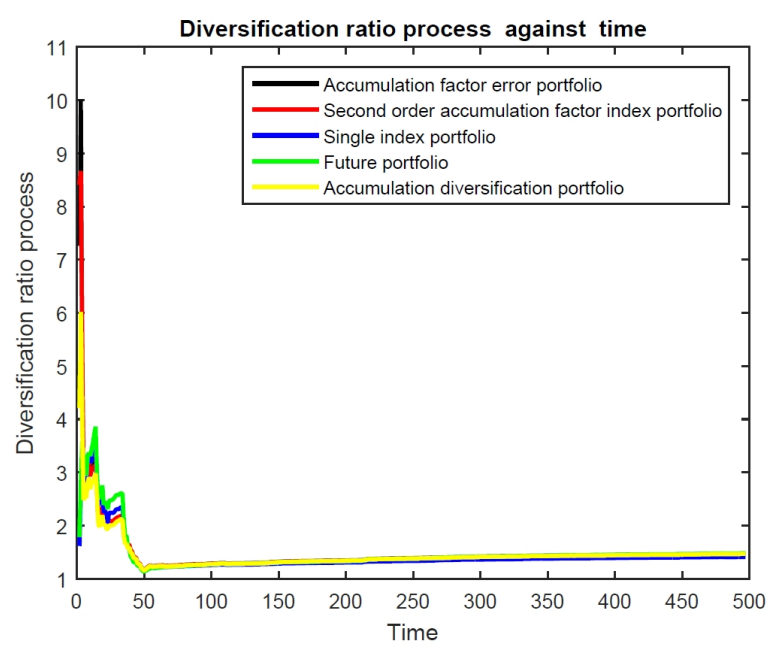

Figure 3. Diversification ratio process of portfolios

Observe from Figure 2 that future portfolio outperforms single index portfolio relative Sharpe ratio process metric. It is clear from the Figure 2 that single index portfolio under performs accumulation factor error portfolio, second order accumulation factor index portfolio, future portfolio and accumulation diversification portfolio relative to Sharpe ratio process. Moreover, accumulation factor error portfolio out performs all portfolio with respect to Sharpe ratio process in the long run. Observe from Figure 3 that future portfolio outperforms single index portfolio relative to diversification ratio process metric except at few instant time points. Furthermore, we note that single index portfolio under performs all other portfolios rela- tive diversification ratio process metric in the long run. Moreover, future portfolio outperforms all other portfolios relative diversification ratio process metric in the long run.

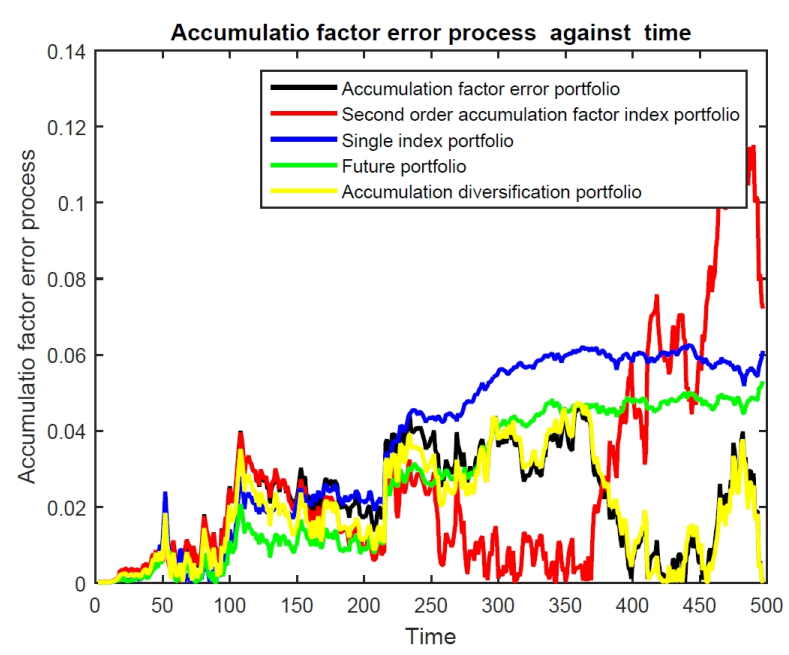

Figure 4. Accumulation factor error process of portfolios

Observe from Figure 4 that future portfolio outperforms single index portfolio relative to accumulation factor error process metric. Clearly, future portfolio outperforms all other portfolios relative to accumulation factor error process metric in the short run.

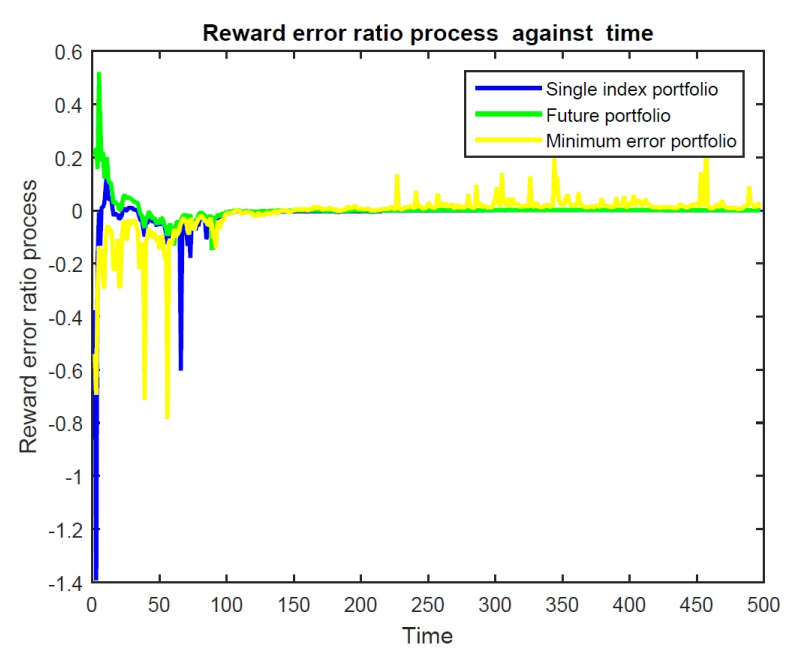

Figure 5. Accumulation factor error process of portfolios

Observe from Figure 5 that future portfolio outperforms single index portfolio relative to reward error ratio process metric except at few instant time points. Clearly, minimum error portfolio outperforms both single index portfolio and future portfolio relative to reward error ratio process metric in the long run. 
Table 1. Summary of total error and total reward error ratio for portfolios

\begin{tabular}{|c|c|c|}
\hline Portfolio & Total error & Total reward error ratio \\
\hline Accumulation factor error portfolio & 10.6583985806473 & 4.39103556060023 \\
\hline Second order accumulation factor index portfolio & 13.2901755489286 & 2.85737884349873 \\
\hline Future portfolio & 13.2281833279834 & 1.19262131494494 \\
\hline Accumulation diversification portfolio & 16.7328157892161 & 16.1342501088985 \\
\hline Single index portfolio & 18.1600826478564 & -6.42598330502002 \\
\hline Minimum error portfolio & 0.993181616376407 & -5.20897041942782 \\
\hline
\end{tabular}

We observe from Table 1 that the best portfolio relative to total error is minimum error portfolio. Again note that the worst portfolio relative to total error metric is single index portfolio. Consider total reward error ratio for each portfolio. The result in Table 1 shows that accumulation diversification portfolio is the best portfolio relative to total reward error ratio metric. Finally, it is clear that future portfolio outperforms single index portfolio relative both total reward error ratio metric and total error metric.

\section{Discussion}

Investors construct equally weighted portfolio when they want to diversify their portfolio. Equally weighted portfolio does not require sophisticated mathematical analysis. Investors can just allocated equal weight for all securities. Researchers have mentioned that mean variance model is realistic for normally distributed returns of securities. However, returns are not normally distributed in practice. Parameters estimation complexity is another problem of mean variance model. Single index model simplifies parameters estimation complexity. Our model, future portfolio model, is better than mean-variance portfolio model with respect to parameters estimation complexity. But it is not better than single index model with respect to parameters estimation complexity. Single index portfolio under performs future portfolio relative accumulation factor error process metric, diversification ratio process metric, Sharpe ratio process metric, total error metric and total reward error ratio metric except at few instant time points. Furthermore, single index portfolio under performs all other portfolios relative Sharpe ratio process metric. Moreover, single index portfolio under performs all other portfolios except second order accumulation factor index portfolio and minimum error portfolio relative accumulation factor error process metric, diversification ratio process metric and Sharpe ratio process metric.

Table 2. Summary of the number of estimated parameters for portfolios

\begin{tabular}{|c|c|c|}
\hline Portfolio & Number of securities & Number of estimated parameters \\
\hline Accumulation factor error portfolio & $n$ & $2 n$ \\
\hline Second order accumulation factor index portfolio & $n$ & $2 n+\frac{n(n+1)}{2}$ \\
\hline Future portfolio & $n$ & $4 n+1$ \\
\hline Accumulation diversification portfolio & $n$ & $3 n$ \\
\hline Single index portfolio & $n$ & $3 n+1$ \\
\hline Mean variance portfolio & $n$ & $n+\frac{n(n+1)}{2}$ \\
\hline
\end{tabular}

The above Table 2 shows that accumulation factor error portfolio is the most simplest portfolio among all portfolios given in Table 2. Observe from Table 2 that second order accumulation factor index portfolio is the most complex portfolio among all portfolios given in Table 2 .

Note that we use market index portfolio return to construct single index portfolio and future portfolio. But we do not use market index portfolio return to construct other portfolio of this study. The two portfolios that consider the effect of market factor on portfolio selection are single index portfolio and future portfolio. Therefore, we realized that future portfolio is better than single index portfolio with respect to portfolio performance measures based on the effect market factor on portfolio selection.

\section{Conclusion and recommendation}

In this research, we reviewed both mean-variance and single index model. Furthermore, we proposed two novel models to construct future portfolio. The first model is future expected return forecasting model. The second model is future portfolio model. We applied capital asset pricing model to estimate portfolio current variance. Moreover, we solved future portfolio model analytically. Finally, we used Sharpe ratio process metric, diversification ratio process metric and accumulation factor error process metric to evaluate the performance of future portfolio relative to single index portfolio. Our empirical result shows that future portfolio outperforms single index portfolio based on Sharpe ratio process metric, diversification ratio process metric and accumulation factor error process metric except at few instant time points. We introduced new portfolio performance measures called accumulation factor error process metric, total error metric and total reward error ratio metric to evaluate the performance of portfolios. Moreover, we constructed future expected return forecast model to forecast securities future expected return. Furthermore, we constructed different portfolio selection models to construct portfolios. In this research, we have constructed future portfolio which considers market factor and other four portfolios which do not consider market factor. Since single portfolio depends on market factor return, it is reasonable compare future portfolio with single index portfolio using portfolio performance measures. The result of this study shows that future portfolio outperforms single index portfolio. However, single index portfolio is better than future portfolio with respect to parameters' estimation complexity.

Now we would like to recommend on this research. In this research, we have not considered the transaction cost for portfolio selection. We assumed that transaction cost is zero. But in practice there is transaction cost. Therefore, we recommend researchers to study the portfolio selection methods by considering transaction cost. We note that there are three portfolios once we construct future portfolio. These are risk free security portfolio, market portfolio and risky portfolio. We recommend researchers to construct complete portfolio by combining risky portfolio, risk free security portfolio and market portfolio.

\section{REFERENCES}

[1] Kolusheva, Daniela, Out-of-sample performance of asset allocation strategies, Available at SSRN 1802491, 2008, doi: 10.2139/ssrn.1802491. 
[2] Ulf, Herold and Raimond, Maurer, Portfolio choice and estimation risk. A comparison of Bayesian to heuristic approaches, ASTIN Bulletin: The Journal of the IAA, Cambridge University Press, vol. 36, no. 1, pp. 135-160, 2006, doi: 10.1017/S0515036100014434.

[3] Lai, Tze Leung and Xing, Haipeng and Chen, Zehao, Mean-variance portfolio optimization when means and covariances are unknown, The Annals of Applied Statistics, Institute of Mathematical Statistics, vol. 5, no. 2A, pp. 798-823, 2011, doi: 10.1214/10-AOAS422.

[4] Gökgöz, Fazil, Mean variance optimization via factor models in the emerging markets: evidence on the Istanbul Stock Exchange, Investment Management and Financial Innovations, vol. 6, no. 3, pp. 43-53, 2009, https : //www.researchgate.net/publication/286323853.

[5] Kamau, Cathrine W, The applicability of the capital asset pricing model and Fama-French three factor model on stocks listed in the Nairobi securities exchange, University of Nairobi, 2014, http : //hdl.handle.net/11295/76596.

[6] Virlics, Agnes, Investment decision making and risk, Procedia Economics and Finance, Elsevier, vol. 6, pp. 169 177, 2013, doi: 10.1016/S2212-5671(13)00129-9.

[7] Hue, B and Jinks, A and Spain, J and Bora, M and Siew, S, Investment risk for long-term investors: risk measurement approaches: Considerations for pension funds and insurers, British Actuarial Journal, Multidisciplinary Digital Cambridge University Press, vol. 24, 2019, doi.org/10.1017/S1357321719000102.

[8] Selmier II, W Travis and Penikas, Henry and Vasilyeva, Ksenia, Financial risk as a good, Procedia Computer Science, Elsevier, vol. 31, 2014, pp. 115-123, doi.org/10.1016/j.procs.2014.05.251.

[9] Khanal, Aditya $\mathrm{R}$ and Mishra, Ashok $\mathrm{K}$ and $\mathrm{Ku}-$ mar, Anjani, Risk Preference and Adoption of Risk Management Strategies: Evidence from High-Value Crop Production in Emerging Economy, 2019 Annual Meeting, July 21-23, Atlanta, Georgia 291285, Agricultural and Applied Economics Association, 2019, doi.org/10.22004/ag.econ.291285.

[10] Sulaiman, Ebrahim Kunju, An empirical analysis of financial risk tolerance and demographic features of individual investors, Procedia Economics and Finance, vol. 2, 2012, pp. 109-115, doi.org/10.1016/S22125671(12)00070-6.

[11] Sulewski, Piotr and Wkas, Adam and Kobus, Pawel and Pogodzinska, Kinga and Szymanska, Magdalena and Sosulski, Tomasz, Farmers' Attitudes towards Risk-An Empirical Study from Poland, Agronomy, Multidisciplinary Digital Publishing Institute, vol. 10, 2020, pp. 1555, doi.org/10.3390/agronomy10101555.
[12] Sutejo, Bertha Silvia and Pranata, Yohanes Kokoh Natan and Mahadwartha, Putu Anom, Demography factors, financial risk tolerance, and retail investors, 15th International Symposium on Management,2018, doi.org/10.2991/insyma-18.2018.20.

[13] Irandoust, Manuchehr, Factors associated with financial risk tolerance based on proportional odds model: Evidence from Sweden, Journal of Financial Counseling and Planning, Springer, vol. 28, 2017, pp. 155-164, doi.org/10.1891/1052-3073.28.1.155.

[14] Duan, Yaoyao Clare, A multi-objective approach to portfolio optimization, Rose-Hulman Undergraduate Mathematics Journal, vol. 8, no. 1, pp. 12, 2007, https://scholar.rose-hulman.edu/rhumj/vol8/iss1/12.

[15] Nguyen, Hoang, Portfolio Optimization Methods: The Mean-Variance Approach and the Bayesian Approach, The University of Mississippi, 2019, http://thesis.honors.olemiss.edu/id/eprint/1398.

[16] Kresta, Aleš and others, Application of Performance Ratios in Portfolio Optimization, Acta Universitatis Agriculturae et Silviculturae Mendelianae Brunensis, Mendel University Press, vol. 63, pp. 1969-1977, 2015, doi.org/10.11118/201563061969.

[17] Petronio, Filomena and Lando, Tommaso and Biglova, Almira and Ortobelli, Sergio, Optimal portfolio performance with exchange traded funds, VŠB (Technical University of Ostrava, Faculty of Economics), 2014, doi.org/10.7327/cerei.2014.03.01.

[18] Rossi, Matteo, The capital asset pricing model: a critical literature review, Global Business and Economics Review,Inderscience Publishers, vol. 18, no. 5, pp. 604-617, 2016, doi.org/10.1504/GBER.2016.078682.

[19] Biglari, Bahram and Nazaripour, Mohammad, The method of stock selection with the intention of portfolio formation, Problems and perspectives in management, Business Perspectives, vol. 14, no. 3, pp. 429-438, 2016, doi.org/10.21511/ppm.14(2-1).2016.18.

[20] Musau, John, The Reliability of Capital Asset Pricing Model on Valuation of Listed Firms at the Nairobi Securities Exchange, University Of Nairobi, 2016, http://hdl.handle.net/11295/97889.

[21] Kourtis, The Sharpe ratio of estimated efficient portfolios, Finance Research Letters, Elsevier, vol. 17, pp. 72-78, 2016, doi.org/10.1016/j.frl.2016.01.009.

[22] Nandan, Tanuj and Srivastava, Nivedita, Construction of Optimal Portfolio Using Sharpe's Single Index Model: An Empirical Study on Nifty 50 Stocks, Journal of Management Research and Analysis, Ip Innovative Publication Pvt. Ltd., vol. 4, no. 2, pp. 74-83, 2017, doi.org/10.2139/ssrn.3259328. 
[23] Singh, Saurabh and Gautam, Jayant, The single index model \& the construction of optimal portfolio: A case of banks listed on NSE India, Risk governance \& control: financial markets \& institutions, vol. 4, no. 2, pp. 110-115, 2014, doi.org 10.22495/rgcv4i2c1art3.

[24] Marhfor, A, Portfolio Performance Measurement: Review of Literature and Avenues of Future Research, American Journal of Industrial and Business Management, vol. 6, no. 4, pp. 432-438, 2016, doi.org10.4236/ajibm.2016.64039.

[25] Heng-Hsing Hsieh, Kathleen Hodnett, A Review of Performance Evaluation Measures for ActivelyManaged Portfolios, Journal of Economics and Behavioral Studies, vol. 5, no. 12, pp. 815-824, 2013, http://hdl.handle.net/10566/2582.

\section{Appendix}

Lemma 7.1 (Accumulation factor constraint lemma). The accumulation factor of portfolio of $n$ securities over period $[2, \tau]$ is defined by:

$$
a_{\tau}=\sum_{j=1}^{n}\left(\prod_{t=2}^{\tau}\left(1+r_{t j}\right)\right) w_{j}=\prod_{t=2}^{\tau} \sum_{j=1}^{n}\left(1+r_{t j}\right) w_{j} .
$$

Proof. Let $P_{c}$ be a portfolio of $n$ securities. Suppose that return of this portfolio $r_{t c}$. Define $w_{j} \sum_{j=1}^{n} p_{1 j} q_{j}=p_{1 j} q_{j}$. Then portfolio value at time $T$ is defined by $v_{\tau}=\sum_{j=1}^{n} p_{\tau j} q_{j}$. Clearly, $v_{\tau}=v_{1} \prod_{t=2}^{\tau}\left(1+r_{t c}\right)$, where $r_{t c}=\sum_{j=1}^{n} r_{t j} w_{j}$ is return of portfolio at time $t$. Similarly, $p_{\tau j}=p_{1 j} \prod_{t=1}^{\tau}\left(1+r_{t j}\right)$. This implies that $v_{\tau}=v_{1} \sum_{j=1}^{n}\left(\prod_{t=2}^{\tau}\left(1+r_{t j}\right)\right) w_{j}$. Clearly, $\sum_{j=1}^{n} w_{j}=1$. Note that $1+r_{t c}=\sum_{j=1}^{n}\left(1+r_{t j}\right) w_{j}$. It follows from $v_{\tau}$ expression that $\sum_{j=1}^{n}\left(\prod_{t=2}^{\tau}\left(1+r_{t j}\right)\right) w_{j}=$ $\prod_{t=2}^{\tau} \sum_{j=1}^{n}\left(1+r_{t j}\right) w_{j}$. Hence the result is obtained.

Lemma 7.2. Let $f_{t}(x)$ be a non-zero differentiable function of $x \forall t=1,2, \ldots, \tau$. Then

$$
\frac{d \prod_{t=1}^{\tau} f_{t}(x)}{d x}=\left(\prod_{t=1}^{\tau} f_{t}(x)\right)\left(\sum_{t=1}^{\tau} \frac{\frac{d f_{t}(x)}{d x}}{f_{t}(x)}\right) .
$$

Proof. Let $u(x)=\prod_{t=1}^{\tau} f_{t}(x)$. Then

$$
\ln (u(x))=\sum_{t=1}^{\tau} \ln \left(f_{t}(x)\right)
$$

Differentiate both sides of equation (50) with respect to $x$.

$$
\frac{\frac{d u(x)}{d x}}{u(x)}=\sum_{t=1}^{\tau} \frac{\frac{d f_{t}(x)}{d x}}{f_{t}(x)}
$$

This implies that

$$
\frac{d u(x)}{d x}=u(x) \sum_{t=1}^{\tau} \frac{\frac{d f_{t}(x)}{d x}}{f_{t}(x)} .
$$

Substitute $u(x)=\prod_{t=1}^{\tau} f_{t}(x)$ in to the equation (52) to find the desired result. Hence the proof followed.
Theorem 7.3 (Portfolio variance approximation theorem). Portfolio variance can be determined approximately by three factors, namely, investment horizon, portfolio expected return and portfolio accumulation factor. Mathematically, this statement is equivalent to the following approximation.

$$
\left(a_{p}-2\right)^{2} \approx \tau\left(\mu_{p}-1\right)^{2}+(\tau-1)\left(\sigma_{p}^{2}-1\right) .
$$

Proof. Apply natural logarithm on both sides of equation (48). Then use second order Taylor series approximation. Define

$$
\begin{gathered}
u_{\tau}=\sum_{j=1}^{n}\left(\prod_{t=1}^{\tau}\left(1+r_{t j}\right)\right) w_{j} \\
v_{\tau}=\prod_{t=1}^{\tau} \sum_{j=1}^{n}\left(1+r_{t j}\right) w_{j} .
\end{gathered}
$$

Thus, we have the following approximation equations.

$$
\begin{gathered}
\ln \left(u_{\tau}\right) \approx u_{\tau}-1-\frac{1}{2}\left(u_{\tau}-1\right)^{2}, \\
\ln \left(v_{\tau}\right) \approx \sum_{t=1}^{\tau}\left(\sum_{j=1}^{n} r_{t j} w_{j}-\frac{1}{2}\left(\sum_{j=1}^{n} r_{t j} w_{j}\right)^{2}\right) .
\end{gathered}
$$

Note that

$$
\sum_{t=1}^{\tau}\left(\sum_{j=1}^{n} r_{t j} w_{j}\right)^{2}=(\tau-1) \sigma_{p}^{2}+\tau \mu_{p}
$$

Clearly,

$$
\sum_{t=1}^{\tau} \sum_{j=1}^{n} r_{t j} w_{j}=\tau \mu_{p}
$$

Substitite equations (58) and (59) in to the equation (57) to get equation (60).

$$
\left.\ln \left(\prod_{t=1}^{\tau} \sum_{j=1}^{n}\left(1+r_{t j}\right) w_{j}\right)=\tau \mu_{p}-\frac{1}{2}\left((\tau-1) \sigma_{p}^{2}+\tau \mu_{p}\right)\right) .
$$

It follows from equations (56) and (60) that

$$
\left.\left(a_{p}-1\right)-\frac{1}{2}\left(a_{p}-1\right)^{2} \approx \tau \mu_{p}-\frac{1}{2}\left((\tau-1) \sigma_{p}^{2}+\tau \mu_{p}\right)\right) .
$$

Equation (61) is equivalent to

$$
\left(a_{p}-2\right)^{2} \approx \tau\left(\mu_{p}-1\right)^{2}+(\tau-1)\left(\sigma_{p}^{2}-1\right) .
$$

Hence the proof followed.

\section{Theorem 7.4.}

$$
\mu_{(t+1) p} \approx \mu_{(t+1) p}^{\delta} .
$$

Proof. It follows from Theorem 7.3 that

$$
\left(a_{(t+1) p}-2\right)^{2} \approx(t+1)\left(\mu_{(t+1) p}-1\right)^{2}+t\left(\sigma_{(t+1) p}^{2}-1\right) .
$$

Define

$$
q z_{1}=(t-1) \sigma_{t p}^{2}+t(t+1)\left(\mu_{(t+1) p}-\mu_{t p}\right)^{2}-t .
$$


Substitute equation (35) in to the equation (64) to get equation (66)

$$
\left(a_{(t+1) p}-2\right)^{2} \approx(t+1)\left(\mu_{(t+1) p}-1\right)^{2}+q z_{1} .
$$

This implies that

$$
\left(a_{(t+1) p}-2\right)^{2} \approx(t+1)\left(\mu_{(t+1) p}^{2}-2 \mu_{(t+1) p}+1\right)+q z_{1} .
$$

Define

$q z_{2}=-2(t+1)\left(1+t \mu_{t p}\right) \mu_{(t+1) p}+1+(t-1) \sigma_{t p}^{2}+t(t+1) \mu_{t p}^{2}$.

It follows from equation (67) that

$$
\left(a_{(t+1) p}-2\right)^{2} \approx(t+1)^{2} \mu_{(t+1) p}^{2}+q z_{2} .
$$

Clearly,

$a_{(t+1) p}=\left(1+r_{(t+1) p}\right) a_{t p}=\left(1+(t+1) \mu_{(t+1) p}-t \mu_{t p}\right) a_{t p}$

This implies that

$$
\left(a_{(t+1) p}-2\right)^{2}=\left(\left((t+1) \mu_{(t+1) p}-t \mu_{t p}\right) a_{t p}+a_{t p}-2\right)^{2} .
$$

\section{Define}

$q z_{3}=\left(a_{t p}-2\right)^{2}+2\left(a_{t p}-2\right)\left(\left((t+1) \mu_{(t+1) p}-t \mu_{t p}\right) a_{t p}\right)$

It follows from equation (71) that

$$
\left(a_{(t+1) p}-2\right)^{2}=\left(\left((t+1) \mu_{(t+1) p}-t \mu_{t p}\right) a_{t p}\right)^{2}+q z_{3} .
$$

Clearly, $2 a_{t p}\left(\left((t+1) \mu_{(t+1) p}-t \mu_{t p}\right) a_{t p}\right) \approx 0$ is a small number. Define

$$
q z_{4}=\left(a_{t p}-2\right)^{2}-4\left(\left((t+1) \mu_{(t+1) p}-t \mu_{t p}\right) a_{t p}\right) .
$$

This implies that

$$
\left(a_{(t+1) p}-2\right)^{2} \approx\left(\left((t+1) \mu_{(t+1) p}-t \mu_{t p}\right) a_{t p}\right)^{2}+q z_{4} .
$$

Define

$$
q z_{5}=\left(a_{t p}-2\right)^{2}+4 t a_{t p} \mu_{t p}-4(t+1) a_{t p} \mu_{(t+1) p} .
$$

Clearly,

$$
\left(a_{(t+1) p}-2\right)^{2} \approx\left(\left((t+1) \mu_{(t+1) p}-t \mu_{t p}\right) a_{t p}\right)^{2}+q z_{5} .
$$

Define

$$
q z_{6}=a_{t p}^{2} t^{2} \mu_{t p}^{2}+\left(a_{t p}-2\right)^{2}+4 t a_{t p} \mu_{t p}
$$

Define

$$
q z_{7}=-2(t+1)\left(2 a_{t p}+t a_{t p}^{2} \mu_{t p}\right) \mu_{(t+1) p} .
$$

It follows from equation (77) that

$$
\left(a_{(t+1) p}-2\right)^{2} \approx a_{t p}^{2}(t+1)^{2} \mu_{(t+1) p}^{2}+q z_{6}+q z_{7} .
$$

Define

$$
\begin{gathered}
x_{2 t}=(t+1)^{2}\left(a_{t p}^{2}-1\right) . \\
x_{1 t}=2(t+1)\left(1+t \mu_{t p}-\left(2 a_{t p}+t a_{t p}^{2} \mu_{t p}\right)\right) .
\end{gathered}
$$

$x_{0 t}=a_{t p}^{2} t^{2} \mu_{t p}^{2}+\left(a_{t p}-2\right)^{2}+4 t a_{t p} \mu_{t p}-1-(t-1) \sigma_{t p}^{2}-t(t+1) \mu_{t p}^{2}$.

It follow from equations (69) and (77) that

$$
x_{2 t} \mu_{(t+1) p}^{2}+x_{1 t} \mu_{(t+1) p}+x_{0 t} \approx 0 .
$$

Clearly,

$$
\left(a_{t p}-2\right)^{2}-(t-1) \sigma_{t p}^{2}-1-t \mu_{t p}^{2} \approx-2 t \mu_{t p}
$$

Substitute equation (85) in to the equation (83) to get equation (86).

$$
x_{0 t} \approx\left(a_{t p}^{2}-1\right) t^{2} \mu_{t p}^{2}+2 t \mu_{t p}\left(2 a_{t p}-1\right) .
$$

Define

$$
c_{0 t}=\left(a_{t p}^{2}-1\right) t^{2} \mu_{t p}^{2}+2 t \mu_{t p}\left(2 a_{t p}-1\right) .
$$

It follows from equations (88) and (86) that

$$
x_{2 t} \mu_{(t+1) p}^{2}+x_{1 t} \mu_{(t+1) p}+c_{0 t} \approx 0 .
$$

Hence the proof followed. 